\title{
Efectos de la temperatura en la resonancia ferromagnética: estudio comparativo para diferentes materiales
}

\author{
Carlos A. Mercado ${ }^{1, *}$, Jorge A. Otalora ${ }^{2}$, Omar J. Suarez ${ }^{1}$ \\ ${ }^{1}$ Departamento de Física, Universidad de Sucre, Sincelejo, Colombia \\ ${ }^{2}$ Institute of Metallic Materials at the Leibniz Institute for Solid State and Materials Research, Dresden, Alemania
}

\begin{abstract}
Resumen
En este trabajo se estudió teóricamente el efecto de la temperatura sobre la frecuencia de resonancia ferromagnética de una partícula magnética anisotrópica; se analizaron los materiales FePt, Co y Ni. El sistema se modeló empleando la ecuación de movimiento de Landau-Lifshitz-Bloch (LLB); se calculó el tensor de susceptibilidad magnética, el cual brinda información de la potencia de absorción y frecuencia de resonancia del sistema. Se encontró que la frecuencia de resonancia experimentó un corrimiento hacia valores más bajos a medida que se incrementaba la temperatura del material. En los materiales de anisotropía más alta, la resonancia se presenta para los campos más bajos. Además, se observó en todos los materiales una disminución en la absorción de energía a medida que aumentó la temperatura. Se pudo concluir que la temperatura y la anisotropía cristalina ejercen una marcada influencia en los valores de campo y en la frecuencia de resonancia, así como en la absorción de energía.
\end{abstract}

Palabras clave: Resonancia ferromagnética; Ecuación de Landau-Lifshitz-Bloch; Anisotropía cristalina.

Effects of temperature on the ferromagnetic resonance of particles: Comparative study for different materials

\begin{abstract}
In this work, we studied from a theoretical approach, the effect of temperature on the frequency of ferromagnetic resonance of an anisotropic magnetic particle; we analyzed the materials FePt, Co, and Ni. Using the LandauLifshitz-Bloch (LLB) equation of motion, we calculated the magnetic susceptibility tensor, which provides information on the absorption power and ferromagnetic resonance of the system. We found that the resonance frequency underwent a shift toward the lower frequency values as the temperature of the material increased. In materials with high anisotropy, the resonance is presented to lower fields. Furthermore, we observed in all materials a decrease in energy absorption as the temperature increased. We concluded that the temperature and the crystalline anisotropy have a strong influence on field values and resonance frequency, as well as on energy absorption.
\end{abstract}

Key words: Crystalline anisotropy; Ferromagnetic resonance; Landau-Lifshitz Bloch equation.

\section{Introducción}

El estudio de las llamadas nanoestructuras magnéticas suscita gran interés debido a sus propiedades físicas, entre las cuales se destacan los bajos puntos de fusión, la fotoluminiscencia debida a efectos cuánticos, y el superparamagnetismo en algunos materiales como el hierro (Fe), el cobalto (Co) y el níquel $(\mathrm{Ni})$, y a sus diversas aplicaciones tecnológicas $(\mathbf{L u}$, et al., 2004; Gupta, et al., 2005; Sun, 2000; Cowburn, et al., 1999; Dormann, et al., 2007; Landeros, et al., 2009; Cisternas \& Vogel, 2015), por ejemplo, los dispositivos de alta frecuencia, los filtros de información, las guías de onda, los cabezales de lectura en discos duros, las memorias magnéticas de acceso aleatorio (magnetoresistive randomaccess memory, MRAM), las MRAM de torsión de espín, y los osciladores de torsión de espín, entre otros (Philip, et al., 2008; Philip, et al., 2003).
Las propiedades magnéticas de nanoestructuras ferromagnéticas como las películas delgadas, los nanohilos, y los nanotubos, entre otras, se han estudiado exhaustivamente tanto de forma experimental (Nielsch, et al., 2005; Schellekens, et al., 2013) como teórica (D Albuquerque \& Castro, et al., 2002; García del Muro \& Solans, 1997; Suarez O. J., et al., 2009; Suarez, et al., 2012). Su estudio teórico generalmente se ha abordado a partir del régimen de temperatura. A temperatura cero la dinámica de la magnetización se ha investigada mediante el uso de las ecuaciones dinámicas de Landau-Lifshitz (LL) y Landau-Lifshitz-

\footnotetext{
*Correspondencia:

Carlos A. Mercado; camv5120@gmail.com

Recibido: 10 de junio de 2019

Aceptado: 22 de julio de 2019

Editor: Jairo Roa Rojas
} 
Gilbert (LLG) (Landau \& Lifshitz, 1935; Mayergoyz, et al., 2009). En el caso de la temperatura finita, la dinámica magnética se ha modelado mediante el uso de la ecuación de Landau-Lifshitz-Bloch (LLB) (Garanin, 1991; Garanin, 1997), con resultados confiables en los fenómenos que se presentan a distintas temperaturas y escalas de tiempo ultrarrápidas. Por ejemplo, el método se ha convertido en una herramienta indispensable para la caracterización de la demagnetización ultrarrápida inducida por láser (Sultan, $\boldsymbol{e t}$ al., 2012), el movimiento de la pared de dominio impulsado térmicamente a través del efecto espín-Seebeck (Hinzke \& Nowak, 2011), el efecto de torsión de giro a temperaturas elevadas (Haney \& Stiles, 2009; Schieback, et al., 2009), la grabación magnética asistida por calor (McDaniel, 2012), la dinámica ultrarrápida en materiales ferrimagnéticos (Atxitia, et al., 2012; Suarez, et al., 2015), y el caos determinista en partículas magnéticas (Suarez, et al., 2017).

En el área de la demagnetización ultrarrápida inducida por láser, se ha demostrado que la ecuación LLB describe adecuadamente la dinámica en materiales como el $\mathrm{Ni}$ y el gadolinio (Gd). La característica principal de la ecuación LLB, que la hace idónea para la dinámica de magnetización ultrarrápida, es que presenta un término de relajación longitudinal que proviene de la fuerte interacción magnón-fonón.

Uno de los efectos más estudiados para entender la dinámica de magnetización de partículas magnéticas ha sido la resonancia ferromagnética (RFM). Esta técnica se ha utilizado para medir parámetros de disipación, la relación giromagnética y la constante de anisotropía en películas delgadas (Farle, 1998; Ellis, et al., 2012) y en sistemas de válvula de espín, compuestas de iridio-manganeso (IrMn), $\mathrm{Co}$, rutenio $(\mathrm{Ru})$ y níquel-hierro $(\mathrm{NiFe})$. Los modelos teóricos se han elaborado con la aproximación de temperatura cero mediante las ecuaciones LL y LLG. En estos estudios se ha observado que la dependencia angular del campo de resonancia muestra un acoplamiento antiferromagnético y campos de anisotropía unidireccionales, lo cual hace que el sistema presente un comportamiento que se ajusta casi a la perfección, en comparación con los resultados experimentales (Tarazona-Coronel, et al., 2014). Asimismo, en múltiples estudios teóricos realizados a temperatura cero se han contrastado sus modelos con los experimentales, con muy buenos resultados (Curiale, 2008; Anaya-Calvo, 2013; Forzani, et al., 2016).

En estudios más completos se han analizado los efectos de la temperatura finita. Por ejemplo, en la caracterización de películas delgadas de $\mathrm{Ni}_{50} \mathrm{Fe}_{50}$ depositadas sobre substratos de $\mathrm{Si}(001)$, se observó mediante el uso de la RFM que el campo de resonancia como función del ángulo del campo en el plano exhibe simetría uniaxial en todas las muestras, con un ligero corrimiento hacia valores menores a medida que disminuye la temperatura. Este comportamiento está asociado con las variaciones térmicas de la magnetización en el plano, lo que a su vez induce una disminución en la anisotropía de superficie en el sistema (Díaz de Sihues, et al., 2006). En estudios teóricos recientes también se han reportado los efectos de la temperatura en la resonancia ferromagnética. Empleando la ecuación LLB, los autores determinaron el espectro de absorción como función de la temperatura en una partícula magnética de material hierro-platino $(\mathrm{FePt})$ y encontraron que la frecuencia de resonancia y el campo aplicado presentaban un corrimiento hacia valores menores de frecuencia con el aumento de la temperatura (Ostler \& Ellis, 2014).

En este trabajo se hizo un estudio teórico sistemático de la resonancia de partículas magnéticas de diferentes materiales ( $\mathrm{FePt}$, Co y $\mathrm{Ni}$ ) a temperatura finita y en presencia de un campo magnético externo. Se escogieron dichos materiales porque se conocen todos los parámetros que se requieren para la modelación bajo la aproximación de campo medio. Son muy pocos los estudios analíticos reportados en las bases de datos sobre nanoestructuras magnéticas en los que se consideran las variaciones de temperatura; ello nos motivó a llevar a cabo diversos estudios en esta línea de trabajo, la cual es un nicho importante de investigación.

El documento está organizado de la siguiente manera: en una primera sección se presenta el modelo teórico, en la siguiente se muestran y analizan los resultados obtenidos y en la última se presentan las conclusiones.

\section{Modelo teórico}

Se estudió una partícula magnética anisotrópica asumida como un monodominio, a temperatura finita en presencia de un campo magnético aplicado con dos componentes: una componente constante, $\mathbf{H}_{0}$, y una componente dependiente del tiempo, $\delta \mathbf{h}(\mathrm{t})$. La evolución temporal del vector de magnetización a temperatura finita se modeló mediante la ecuación de Landau-Lifshitz-Bloch (LLB):

$$
\dot{m}=-\gamma m \times H_{e f f}+\frac{\gamma \alpha_{\|}}{m^{2}}\left(m \cdot H_{e f f}\right) m-\frac{\gamma \alpha_{\perp}}{m^{2}}\left(m \times m \times H_{e f f}\right),
$$

donde $\boldsymbol{m}$ representa el vector de magnetización normalizado $\left(\boldsymbol{m}=\boldsymbol{M} / M_{s}\right) ; M_{s}$ es la magnetización de saturación de la partícula a temperatura cero absoluto; $\alpha_{\|}$y $\alpha_{\perp}$ son los parámetros de disipación longitudinal y perpendicular, respectivamente; $\gamma$ representa la relación de radio giromagnética de un electrón libre, y $\boldsymbol{H}_{\text {eff }}$ representa el campo magnético efectivo del sistema. Este campo contiene todas las contribuciones de campo que sean consideradas en un sistema particular de estudio.

En este caso, el campo efectivo utilizado se expresa de la siguiente forma:

$$
\boldsymbol{H}_{e f f}=\boldsymbol{H}_{A p}+\boldsymbol{H}_{A N}+\frac{1}{2 \widetilde{\chi \|}}\left(1-\frac{m^{2}}{m_{e}^{2}}\right)
$$

donde $\boldsymbol{H}_{A p}$ representa el campo magnético aplicado, y $\boldsymbol{H}_{A N}$ es el campo de anisotropía definido de la forma:

$$
\boldsymbol{H}_{A N}=\frac{-\left(m_{x} \hat{\boldsymbol{x}}+m_{y} \hat{\boldsymbol{y}}\right)}{\widetilde{\chi} \|}
$$

El último término en la ecuación (2) es un campo de temperatura, el cual siempre se encuentra presente en el campo efectivo debido a que es un término que aparece en la deducción de la ecuación LLB. En las ecuaciones (2) y 
(3) $m_{e}(T)$ es la magnetización de equilibrio, y $\tilde{\chi}_{\|}$y $\tilde{\chi}_{\perp}$ son las susceptibilidades magnéticas paralela y perpendicular, las cuales son funciones de la temperatura. Los parámetros $\tilde{\chi}_{\|}, \tilde{\chi}_{\perp}$ y $m_{e}$ se toman desde la aproximación de campo medio. La magnetización de equilibrio se obtiene a partir de la ley de Curie-Weiss: $\mathcal{M}=B\left[\beta\left(\mathcal{M} J_{0}+\mu M_{0}\right)\right]$, donde $\mu$ es el momento magnético atomístico, $J_{0}$ la intensidad del campo de intercambio, y $\beta=1 / k_{B} T$ siendo $k_{B}$ la constante de Boltzmann (Garanin, 1997).

La función $B(y)=$ coth $(y)-1 / y$ representa la función de Langiven, la cual describe la magnetización para materiales como el $\mathrm{Ni}$ y $\mathrm{FePt}$, mientras que para $\mathrm{Co}$, la magnetización se modela con la función de Brillouin: $B(y)$ $=\left(\frac{2 s+1}{2 s}\right) \operatorname{coth}\left(\frac{2 s+1}{2 s} y\right)-\left(\frac{1}{2 s}\right) \operatorname{coth}\left(\frac{1}{2 s} y\right)$, con valor de espín $\mathrm{S}=1 / 2$ (Moreno, et al., 2016).

Los parámetros de disipación longitudinal y perpendicular dados en la ecuación (1) son funciones de la temperatura, que en la aproximación de campo medio se definen como:

$$
\begin{gathered}
\alpha_{\|}=\alpha \frac{2 T}{3 T_{c}} \\
\alpha_{\perp}=\alpha\left(1-\frac{T}{3 T_{c}}\right)
\end{gathered}
$$

donde $\alpha$ es el coeficiente de disipación a temperatura cero. Las susceptibilidades magnéticas paralela y perpendicular son dadas por las expresiones:

$$
\begin{gathered}
\widetilde{\chi} \|(T)=\left(\frac{\mu}{J_{0}}\right) \frac{\beta J_{0} B^{\prime} \mid H_{0}=0}{1-\beta J_{0} B^{\prime} \mid H_{0}=0} \\
\widetilde{\chi_{\perp}}(T)=\frac{M_{s} m_{e}(T),}{(2 K(T)},
\end{gathered}
$$

donde $K(T)=K_{0} m_{e}^{\eta}(T)$, siendo $K_{0}=K(T=0)$ la constante de anisotropía a temperatura cero y $\eta$ un exponente de escala específico para cada material.

Se hizo aquí un estudio comparativo de la resonancia ferromagnética a temperatura finita en tres materiales: FePt, Co y Ni. En la Tabla 1 se presentan los valores de los parámetros utilizados para las simulaciones, así como las correspondientes expresiones para el cálculo de la intensidad del campo de intercambio $\left(\mathrm{J}_{0}\right)$ en la aproximación de campo medio.

\section{Análisis y resultados}

Se estudió una partícula magnética con anisotropía uniaxial a lo largo del eje z; el campo externo aplicado tuvo una componente constante, $\mathbf{H}_{\mathrm{z}}=\mathbf{H}_{0} \| \hat{z}$, aplicada a lo largo del eje fácil y una componente armónica dependiente del tiempo, $\delta \mathbf{h}(t)$, aplicada de forma perpendicular a este con la condición $|\delta \mathbf{h}(\mathrm{t})|<\left|\mathbf{H}_{z}\right|$. Cerca de la resonancia, el campo $\delta \mathbf{h}(\mathrm{t})$ suministra energía suficiente al sistema como para compensar las pérdidas de energía asociadas con la interacción de los momentos magnéticos y el medio circundante, lo cual mantiene la magnetización en un estado dinámico. Dada la excitación del campo dependiente del tiempo, la magnetización puede escribirse como $\mathbf{M}=\mathbf{M}_{0}$ $+\delta \mathbf{m}$, donde $\mathbf{M}_{0}=\mathbf{M}_{0} \boldsymbol{m}$ representa la magnetización de equilibrio y $\delta \mathbf{m}$ representa la parte dinámica de la magnetización, con la condición $|\delta \mathbf{m}|<<\left|\mathbf{M}_{0}\right|$.

Empleando la ecuación LLB, se determinó la evolución temporal del vector de perturbación $\delta \mathbf{m}$, el cual se escribe finalmente en forma matricial como $\delta \mathbf{m}=\tilde{\chi} \delta \mathbf{h}$, donde $\tilde{\chi}$ es un tensor en variable compleja llamado "tensor de susceptibilidad magnética", el cual es dado como $\tilde{\chi}=\gamma \mu_{0} M_{0} m A^{-1}$, donde $\mu_{0}$ es la permeabilidad magnética del vacío, y $\mathbf{A}$ es una matriz $2 \times 2$ cuyas componentes están dadas por:

$$
\begin{aligned}
& -A_{x x}=\Omega_{0}-\frac{i \Omega \alpha_{\perp}}{m^{2}} \\
& \text { - } A_{y x}=i \Omega+\frac{\gamma \alpha_{\|}}{m^{2}}\left(H_{z}+\xi\right) \\
& \text { - } A_{x y}=-i \Omega-\frac{\gamma \alpha_{\|}}{m^{2}}\left(H_{z}+\xi\right) \\
& \text { - } A_{y y}=\Omega_{0}-\frac{i \Omega \alpha_{\perp}}{m^{2}},
\end{aligned}
$$

donde $\Omega_{0}=\gamma\left(H_{z}+\frac{m(T)}{\bar{\chi}_{\perp}}\right)$ y $\xi=\frac{1}{2 \bar{\chi}_{\|}}\left(1-\frac{m^{2}}{m_{e}^{2}}\right)$, siendo $\Omega_{0}$ la frecuencia natural del sistema. La parte imaginaria de una componente del tensor de susceptibilidad magnética brinda información sobre la absorción energética que presenta el sistema. La componente $\mathrm{x}$ de dicho tensor, en nuestro caso, viene dada por la expresión:

$$
\tilde{\chi}_{x x}=\frac{\gamma \mu_{0} M_{s} m(T)\left(\left(\Omega_{0}{ }^{2}-\Omega^{2}\right)+2 i \Omega \Gamma\right)\left(\Omega_{0}-\frac{i \Omega \alpha_{\perp}}{m(T)}\right)}{\left(\Omega_{0}{ }^{2}-\Omega^{2}\right)^{2}+4 \Omega^{2} \Gamma^{2}},
$$

donde $\Gamma=\frac{\Omega_{0} \alpha_{\perp}}{m(T)}-\gamma \alpha_{\|}\left(\frac{H_{Z}}{m(T)}+\xi\right)$.

En las Figuras 1 y 2 se presentan gráficos de la componente $\mathrm{x}$ de la parte imaginaria del tensor de susceptibilidad magnética como función del campo constante $\mathrm{H}_{\mathrm{z}}$ y la frecuencia $\Omega$, respectivamente, para los materiales A) FePt, b) $\mathrm{Co}$ y c) Ni, a diferentes temperaturas. En los diferentes materiales la resonancia se obtiene para diferentes valores del campo aplicado $\mathrm{H}_{\mathrm{z}}$ y la frecuencia del campo dependiente del tiempo.

En la Figura 1 se observa que en materiales con anisotropías más altas, el efecto de la resonancia empieza a evidenciarse a frecuencias y campos más altos, como son los

Tabla 1. Parámetros utilizados para la caracterización del sistema (Anaya-Calvo, 2013; Ostler \& Ellis, 2014; Garanin, 1991).

\begin{tabular}{lcccccc}
\hline Material & \multicolumn{5}{c}{ Parametros } \\
\cline { 2 - 7 } & $\mathbf{M}_{\mathbf{s}}\left(\mathbf{J} / \mathbf{T m}^{\mathbf{3}}\right)$ & $\mathbf{T}_{\mathbf{c}}(\mathbf{K})$ & $\mathbf{K}_{\mathbf{0}}\left(\mathbf{J} / \mathbf{m}^{\mathbf{3}}\right)$ & $\boldsymbol{\mu}\left(\mathbf{A} \mathbf{m}^{\mathbf{2}}\right)$ & $\mathbf{J}_{\mathbf{0}}$ & 3,1 \\
\hline $\mathrm{FePt}$ & $1,05 \times 10^{6}$ & 660 & $8,2 \times 10^{6}$ & $3,23 \boldsymbol{\mu}_{\mathrm{B}}$ & $3 k_{B} T_{c}$ \\
$\mathrm{Co}$ & $1,42 \times 10^{6}$ & 1360 & $5,3 \times 10^{5}$ & $1,72 \boldsymbol{\mu}_{\mathrm{B}}$ & 3 & $k_{B} T_{c}$ \\
$\mathrm{Ni}$ & $4,8 \times 10^{5}$ & 630 & $5,3 \times 10^{3}$ & $0,61 \boldsymbol{\mu}_{\mathrm{B}}$ & 3 & $3 k_{B} T_{c}$ \\
\hline
\end{tabular}


casos del FePt (Figura 1a) y del Co (Figura 1b), donde las frecuencias que se requieren para que se presente el efecto de la resonancia son del orden de $10^{2} \mathrm{GHz}$ y $10 \mathrm{GHz}$. El Ni presenta la menor anisotropía, por lo cual la resonancia se presenta a partir de frecuencias mucho más bajas, del orden de los $10^{\circ} \mathrm{GHz}$ (Figura 1c). El efecto de la temperatura juega un papel importante en la resonancia ferromagnética. Al mantener fija la frecuencia $\Omega$, y al aumentar paulatinamente la temperatura del material, la magnitud del vector de magnetización va disminuyendo, al igual que la anisotropía, por lo que para evidenciar el efecto de la resonancia se requieren valores cada vez más altos del campo $\mathrm{H}_{z}$. La absorción de energía para los diferentes materiales también se ve afectada por la temperatura.

A medida que la temperatura aumenta, la absorción para cada material disminuye, como se evidencia en la altura de los picos de resonancia. En la Figura 1b, para el Co a bajas temperaturas se observa un comportamiento un poco diferente: la absorción desde $\mathrm{T} / \mathrm{T}_{\mathrm{C}}=0$ aumenta hasta una temperatura del orden de $4,5 \mathrm{~T}_{\mathrm{C}}$, a partir de la cual empieza
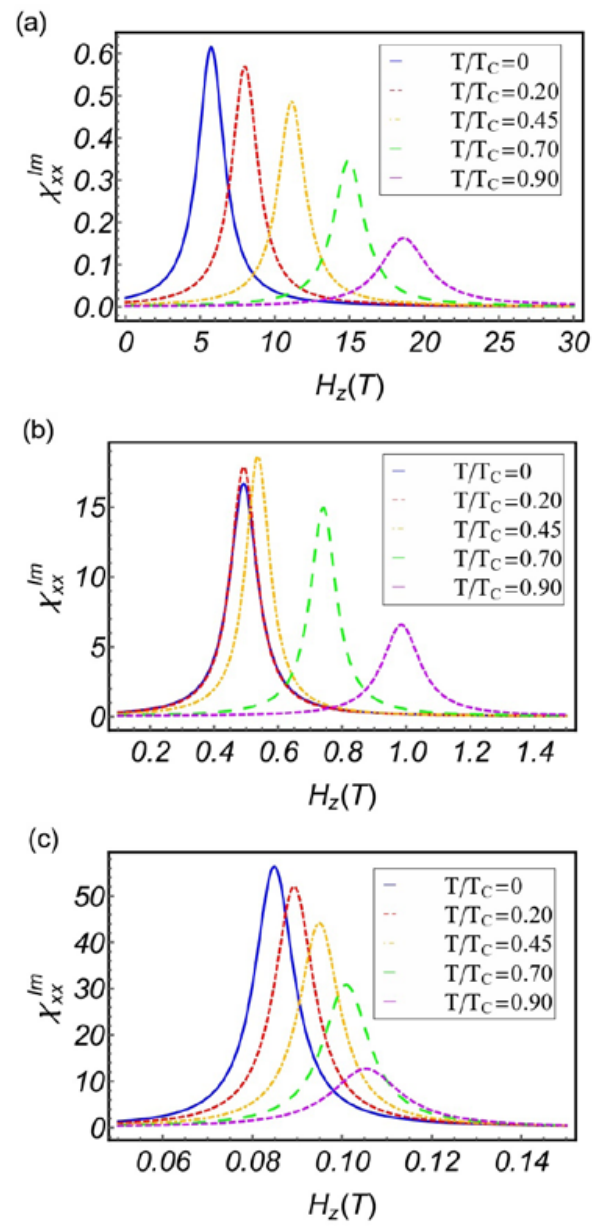

Figura 1. Componente $\mathrm{x}$ de la parte imaginaria del tensor de susceptibilidad magnética como función del campo aplicado $\mathrm{H}_{\mathrm{z}}$ para diferentes temperaturas. Las figuras se realizaron para: (a) FePt a $600 \mathrm{GHz}$, (b) Co a $30 \mathrm{GHz}$ y (c) Ni a $3 \mathrm{GHz}$. a disminuir. Este comportamiento se debe al modelo que se emplea para describir la magnetización de equilibrio, $\mathrm{m}_{\mathrm{e}}$. Con este modelo, $\mathrm{m}_{\mathrm{e}}$ permanece casi constante hasta una temperatura de $0,4 \mathrm{~T}_{\mathrm{C}}$, disminuyendo muy poco hasta una temperatura de $0,5 \mathrm{~T}_{\mathrm{C}}$, lo cual hace que el factor $\Omega_{0} / m(T)$ en el denominador del tensor de susceptibilidad se vea fuertemente afectado.

En la Figura 2 se muestra el gráfico de la componente $\mathrm{x}$ de la parte imaginaria del tensor de susceptibilidad como función de la frecuencia y para diferentes temperaturas. Se puede evidenciar que la frecuencia de resonancia para los diferentes materiales disminuye a medida que aumenta la temperatura del sistema, pero dicha disminución se presenta de manera más marcada en materiales con mayor anisotropía.

En la Figura 3 se muestran los gráficos de la componente $\mathrm{x}$ de la parte imaginaria del tensor de susceptibilidad como función de la amplitud de campo $\mathrm{H}_{\mathrm{z}}$ a una frecuencia $\Omega$ de

(a)

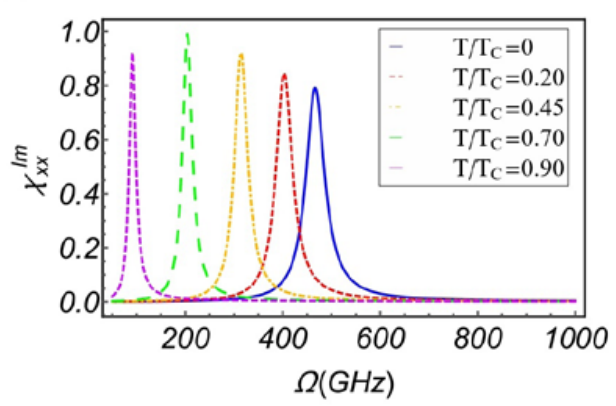

(b)

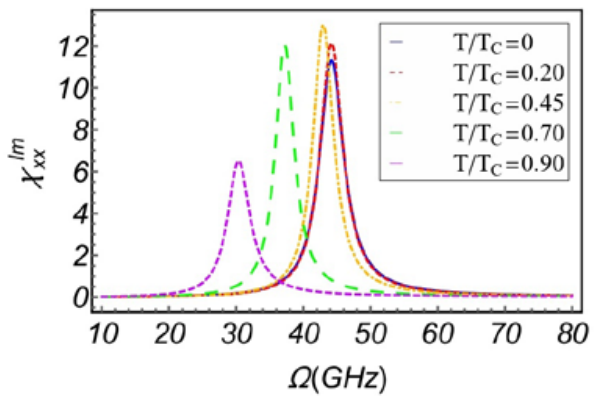

(c)

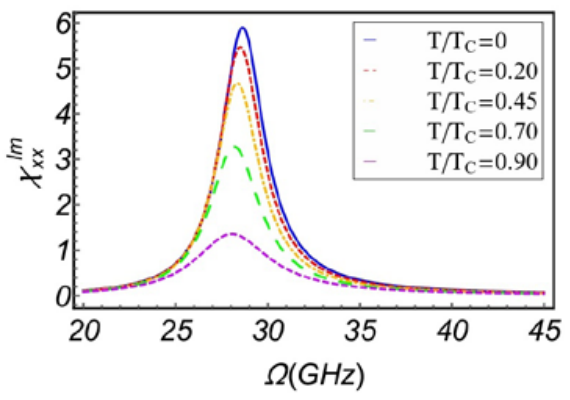

Figura 2. Componente $\mathrm{x}$ de la parte imaginaria del tensor de susceptibilidad magnética como función de la frecuencia para diferentes temperaturas. Las figuras se realizaron para un campo aplicado fijo de 1T en (a) FePt, (b) Co y (c) Ni. 
(a)

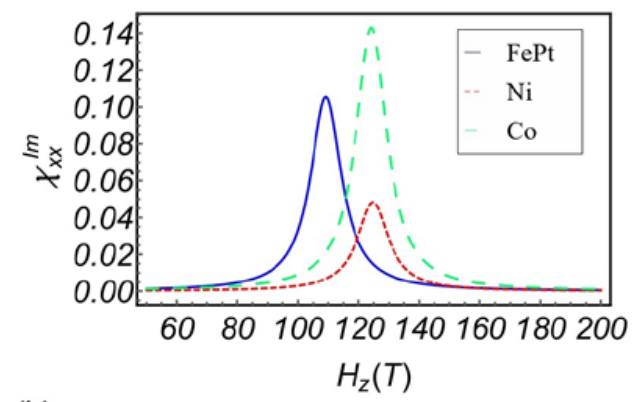

(b)

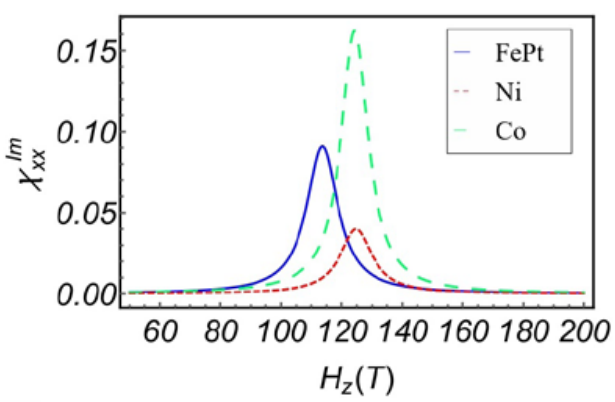

(c)

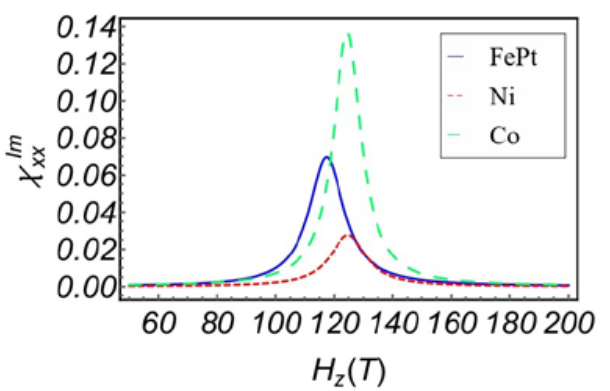

Figura 3. Componente $\mathrm{x}$ de la parte imaginaria del tensor de susceptibilidad magnética como función del campo aplicado para los tres materiales. Las figuras se realizaron para: (a) $\mathrm{T}=0$, (b) $\mathrm{T}=$ $0.4 \mathrm{Tc}$ y (c) $\mathrm{T}=0.7 \mathrm{Tc}$, todos a una frecuencia $\Omega$ de $3500 \mathrm{GHz}$.

$3500 \mathrm{GHz}$ para diferentes temperaturas. En este caso de frecuencias altas para los materiales $\mathrm{Ni}$ y $\mathrm{Co}$, se presentan campos de resonancia mucho más altos que los que presenta el material FePt, el cual posee mayor campo de anisotropía.

La absorción de energía en cada material es diferente: en los materiales Ni y Co se observó que la absorción de energía es diferente en los regímenes de bajas y altas frecuencias. Con bajas frecuencias, como en el caso de la Figura 1, estos materiales presentan mucha más absorción que el material $\mathrm{FePt}$, pero en el régimen de altas frecuencias, la absorción de energía disminuye sustancialmente, como se observa en la Figura 3. Además, en el régimen de altas frecuencias $(\mathrm{Co}, \mathrm{Ni})$, con el aumento de la temperatura en el material FePt el campo de resonancia cambia apreciablemente hacia valores más altos, lo que no se presenta en los materiales $\mathrm{Co}$ y $\mathrm{Ni}$, con los cuales estos campos cambian muy poco.

\section{Conclusiones}

Se analizó teóricamente la resonancia ferromagnética de una partícula magnética anisotrópica a temperatura finita, específicamente con los materiales FePt, Co y Ni. El estudio se hizo empleando la ecuación de Landau-Lifshitz-Bloch. Se encontró que la temperatura tiene un efecto importante en los valores de campo y frecuencia de resonancia. En el régimen de baja frecuencia con el aumento de la temperatura en los tres materiales se presentó un corrimiento en el campo de resonancia hacia valores más altos, acompañado con una disminución en la absorción de energía. En el régimen de alta frecuencia para los materiales $\mathrm{Co}$ y $\mathrm{Ni}$, el campo de resonancia no cambió mucho con el aumento de la temperatura, en tanto que en el material FePt el campo resonante aumentó sustancialmente.

En materiales con alta anisotropía el efecto de la resonancia se evidencia solo con valores altos de campo y frecuencia, como es el caso del material FePt. En los materiales con anisotropía más baja, Co y Ni, la resonancia empieza a observarse a valores bajos de campo y frecuencia. En el caso de los materiales Co y Ni se observaron dos regímenes de resonancia a bajas y altas frecuencias. A altas frecuencias en estos materiales se requieren campos resonantes muy altos, incluso más altos que el requerido en el caso del material FePt, que posee una alta anisotropía. Una buena comprensión de los efectos de la temperatura y de la anisotropía cristalina en las propiedades resonantes de sistemas magnéticos es crucial para todas las potenciales aplicaciones tecnológicas en que se requiera la inyección y la absorción de energía.

\section{Agradecimientos}

Carlos A. Mercado agradece a la Universidad de Sucre por permitir el uso de equipos de cómputo para la realización de los gráficos y las figuras del artículo.

\section{Contribución de los autores}

Carlos A. Mercado: escritura, revisión y elaboración de tablas y figuras del manuscrito. Omar J. Suarez y Jorge A. Otálora: escritura, revisión y corrección del manuscrito.

\section{Conflicto de intereses}

Los autores declaran no tener conflictos de intereses.

\section{Referencias}

Anaya-Calvo J. (2013). Transporte térmico y caracterización RAMAN de nanohilos semiconductores de silicio y siliciogermanio. (Tesis Doctoral). Programa de Doctorado en ciencias Físicas, Universidad de Valladolid. Doi: 10.13140/ RG.2.1.4775.1760

Atxitia U., Nieves P., and Chubykalo-Fesenko O. (2012). LandauLifshitz-Bloch equation for ferrimagnetic materials. Phys. Rev. B 86: 104414. Doi: 10.1103/PhysRevB.86.104414

Cisternas E. \& Vogel E. E. (2015). Improving information storage by means of segmented magnetic nanowires. J. Magn. Magn. Mater. 388: 35-39. Doi: 10.1016/j.jmmm.2015.04.020 
Cowburn R. P., Koltsov D. K., Adeyeye A. O., Welland M. E., and Tricker D. M. (1999). Single-Domain Circular Nanomagnets. Phys. Rev. Lett. 83: 1042. Doi: 10.1103/ PhysRevLett.83.1042

Curiale C. J. (2008). Nanohilos y nanotubos magnéticos. Preparación, caracterización microestructural y estudio de las propiedades eléctricas y magnéticas. (Tesis Doctoral). Instituto Balseiro, Universidad Nacional de Cuyo. Fecha de consulta: 28 de mayo de 2019. Disponible en: https:// www.researchgate.net/publication/43694407_Nanohilos_y_ nanotubos_magneticos_Preparacion_caracterizacion_ microestructural_y_estudio_de_las_propiedades_ electricas y magneticas

d'Albuquerque e Castro J., Altbir D., Retamal J. C., and Vargas P. (2002). Scaling Approach to the Magnetic Phase Diagram of Nanosized Systems. Phys. Rev. Lett. 88: 237202. Doi: 10.1103/PhysRevLett.88.237202

Díaz de Sihues M., Silva P.J., Fermín J.R., Azevedo A., Rezende S.M., and De Aguiar F.M. (2006). Efecto de la temperatura en la resonancia ferromagnética del $\mathrm{Ni} 50 \mathrm{Fe} 50 / \mathrm{Si}(001)$. Rev. Mex. Fís. S52 (3): 143-146. Dispo-nible en: http://www. scielo.org.mx/scielo.php?script=sci_arttext\&pid=S0035001X2006000900041

Dormann J. L., Fiorani D., and Tronc E. (2007). Magnetic Relaxation in Fine-Particle Systems. Advances in Chemical Physics (Wiley, Hoboken, NJ), pp. 283-494. Doi: 10.1002/9780470141571.ch4

Ellis M. O. A., Ostler T. A., and Chantrell R. W. (2012). Classical spin model of the relaxation dynamics of rare-earth doped permalloy. Phys. Rev. B 86: 174418. Doi: 10.1103/ PhysRevB.86.174418

Farle, M. (1998). Ferromagnetic resonance of ultrathin metallic layers. Rep. Prog. Phys. 61: 755. Doi: 10.1088/00344885/61/7/001

Forzani L., Gennaro A.M., Bonin C.J., and Koropecki R.R. (2016). Propiedades magnéticas de nanohilos de níquel en matrices de alúmina porosa. Congreso Internacional de Metalurgia y Materiales SAM-CONAMET. Fecha de consulta: 1 de junio de 2019. Disponible en: http:// conferencias.unc.edu.ar/index.php/sam2016/samconamet2016/paper/viewFile/3030/1272

Garanin, D. A. (1991). Generalized Equation of motion for a ferromagnet. Physica. A 172: 470. Doi: 10.1016/03784371(91)90395-S

Garanin, D. A. (1997). Fokker-Planck and Landau-Lifshitz-Bloch equations for classical ferromagnets. Phys. Rev. B 55: 3050. Doi: 10.1103/PhysRevB.55.3050

García del Muro y Solans, M. (1997). Propiedades magnéticas de materiales nanoestructurados: vidrios metálicos recristalizados y partículas pequeñas de hexaferrita. (Tesis Doctoral). Departamento de Física, Universidad de Barcelona. Fecha de consulta: 2 de junio de 2019. Disponible en: https://www.tdx.cat/handle/10803/1799

Gupta A. K., and Gupta M. (2005). Synthesis and surface engineering of iron oxide nanoparticles for biomedical applications. Biomaterials. 26 (18): 3995-4021. Doi: 10.1016/j.biomaterials.2004.10.012

Haney, P. M. \& Stiles M. D. (2009). Magnetic dynamics with spin-transfer torques near the Curie temperature. Phys. Rev. B 80: 094418. Doi: 10.1103/PhysRevB.80.094418
Hinzke, D. \& Nowak U. (2011). Domain Wall Motion by the Magnonic Spin Seebeck Effect. Phys. Rev. Lett. 107: 027205. Doi: 10.1103/PhysRevLett.107.027205

Landau, L. D. \& Lifshitz, E. M. (1935). On the Theory of the Dispersion of Magnetic Permeability in Ferromagnetic Bodies. Phys. Z. Sowjetunion. 8: 153, reproducido en Collected Papers of L. D. Landau, editado por D. ter Haar (Pergamon, New York), p. 101 (1965). Doi: 10.1016/B9780-08-036364-6.50008-9

Landeros P., Suarez O. J., Cuchillo A., and Vargas P. (2009). Equilibrium states and vortex domain wall nucleation in ferromagnetic nanotubes. Phys. Rev. B 79: 024404. Doi: 10.1103/PhysRevB.79.024404

Lu A.H., Schmidt W., Matoussevitch N., Bönnemann H., Spliethoff B., Tesche B., Bill E., Kiefer W., and Schüth F. (2004). Nanoengineering of a Magnetically Separable Hydrogenation Catalyst. Angewandte Chemie International Edition. 43 (33): 4303-4306. Doi: 10.1002/anie.200454222

Mayergoyz I. D., Bertotti G., and Serpico C. (2009). Nonlinear Magnetization Dynamics in Nanosystems. Napoli, Italia: Elsevier Science. Fecha de consulta: 1 de junio de 2019. Disponible en: https://www.elsevier.com/books/ nonlinear-magnetization-dynamics-in-nanosystems/ mayergoyz/978-0-08-044316-4

McDaniel, T. W. (2012). Application of Landau-Lifshitz-Bloch dynamics to grain switching in heat-assisted magnetic recording. J. Appl. Phys. 112: 013914. Doi: https://doi. org/10.1063/1.4733311

Moreno R., Evans R. F. L., Khmelevskyi S., Muñoz M. C., Chantrell R. W., and Chubykalo-Fesenko O. (2016). Temperature-dependent exchange stiffness and domain wall width in Co. Phys. Rev. B 94: 104433. Doi: 10.1103/ PhysRevB.94.104433

NielschK., Castaño F. J., Ross C. A., and Krishnan R. (2005). Magnetic properties of template synthesized cobalt/polymer composite nanotubes. J. Appl. Phys. 98: 034318. Doi: $10.1063 / 1.2005384$

Ostler, T. A. \& Ellis, M. O. A. (2014). Temperature-dependent ferromagnetic resonance via the Landau-Lifshitz-Bloch equation: Application to FePt. Phys. Rev. B 90: 094402. Doi: 10.1103/PhysRevB.90.094402

Philip J., Shima P. D., and Raj B. (2008). Nanofluid with tunable thermal properties. Applied Physics Letters. 92: 043108. Doi: $10.1063 / 1.2838304$

Philip J., Jaykumar T., Kalyanasundaram P., and Raj B. (2003). A tunable optical filter. Measurement Science \& Technology. 14: 1289-1294. Doi: 10.1088/09570233/14/8/314

Schieback C., Hinzke D., Kläui M., Nowak U., and Nielaba P. (2009). Temperature dependence of the current-induced domain wall motion from a modified Landau-LifshitzBloch equation. Phys. Rev. B 80: 214403. Doi: 10.1103/ PhysRevB.80.214403

Schellekens A. J., Deen L., Wang D., Kohlhepp J. T., Swagten H. J. M., and Koopmans B. (2013). Determining the Gilbert damping in perpendicularly magnetized $\mathrm{Pt} / \mathrm{Co} / \mathrm{AlOx}$ films. Appl. Phys. Lett. 102: 082405. Doi: 10.1063/1.4794538

Suarez O. J., Nieves P., Laroze D., Altbir D., and ChubykaloFesenko O. (2015). Ultrafast relaxation rates and reversal time in disordered ferrimagnets. Phys. Rev. B 92: 144425. Doi: 10.1103/PhysRevB.92.144425 
Suarez O. J., Laroze D., Martínez-Mardones J., Altbir D., and Chubykalo-Fesenko O. (2017). Chaotic dynamics of a magnetic particle at finite temperature. Phys. Rev. B 95: 014404. Doi: 10.1103/PhysRevB.95.014404

Suarez O. J., Vargas P., and Vogel E. E. (2009). Energy and force between two magnetic nanotubes. J. Magn. Magn. Mater. 321: 3658-3664. Doi: 10.1016/j.jmmm.2009.07.009

Suarez, O. J., Pérez, L. M., Laroze, D., and Altbir, D. (2012). Magnetostatic interactions in cylindrical nanostructures with non-uniform magnetization. J. Magn. Magn. Mater. 324: 1698-1705. Doi: 10.1016/j.jmmm.2011.12.032

Sultan M., Atxitia U., Melnikov A., Chubykalo-Fesenko O., and Bovensiepen U. (2012). Electron- and phonon-mediated ultrafast magnetization dynamics of Gd(0001). Phys. Rev. B 85: 184407. Doi: 10.1103/PhysRevB.85.184407

Sun, S. (2000). Monodisperse FePt nanoparticles and ferromagnetic FePt nanocrystal superlattices. Science 287, 1989. Doi: 10.1126/science.287.5460.1989

Tarazona-Coronel H. S., Landauro C. V. and QuispeMarcatoma J. (2014). Resonancia ferromagnética en válvulas de espín: el caso del sistema $\operatorname{IrMn}(150 \AA)$ / $\mathrm{Co}(50 \AA) / \mathrm{Ru}(32 \AA) / \mathrm{NiFe}(50 \AA)$. Revista de Investigación de Física. 17: 141702101. https://www.researchgate.net/ publication/326391354_Resonancia_ferromagnetica_ en_valvulas_de_espin_el_caso_del_sistema IrMn150ACo50ARu32ANiFe50A 